\title{
OS EFEITOS DA UTILIZAÇÃO DA MÚSICA PARA OS IDOSOS: REVISÃO SISTEMÁTICA
}

\author{
Lorena Gomes Enfermeira graduada pela Escola Bahiana de \\ Medicina e Saúde Pública \\ Juliana Bezerra do Amaral Professora Assistente da Escola Bahiana de \\ Medicina e Saúde Pública. Doutora em \\ Enfermagem pelo Programa de Pós Graduação \\ em Enfermagem da Universidade Federal da \\ Bahia
}

\begin{abstract}
Resumo
Esta pesquisa teve por objetivo traçar o perfil e analisar as publicações acerca do uso da música como ferramenta no cuidado à pessoa idosa. A revisão foi realizada nas bases Scielo, Capes e Lilacs a partir do descritor subjetivo idoso. Foram selecionados artigos publicados em periódicos indexados no período de 2000 a 2011 . A partir dos critérios de inclusão/exclusão foram recuperados 05 trabalhos, na íntegra, para discussão. Estes critérios relativos á área de saúde acometem diretamente os benefícios que a utilização da música provoca na saúde e na vida do idoso, evocando juntamente noções de bem-estar, qualidade de vida, valorização e prazer de viver. Os trabalhos abordam a conceitualização do uso direto da música na vida do idoso. Concluindo, os resultados confirmam a atualidade e pertinência do tema para uso na promoção da saúde na população idosa, alertando a necessidade de maiores investigações relacionadas à prática de tal intervenção pelos profissionais de saúde.
\end{abstract}

Palavras-chaves: Música; Idosos e saúde.

\section{THE EFFECTS OF THE USE OF MUSIC FOR THE ELDERLY: SYSTEMATIC REVIEW}

\begin{abstract}
This study aimed to profile publications concerning music usage in the lives of the elderly. To this goal we have considered scientific studies on the quality of life of the elderly and discussed the impact of music as health promoter. This review was conducted in the Scielo, Lilacs and Capes databases searching for the subjective descriptor 'elderly'. We have selected articles published in journals indexed between 2000 and 2011. Based on the criteria of inclusion / exclusion we have recovered 5 articles which were studied. Most are related to the area of health and medicine. Most of these only address the direct benefits music has on the health and life of the elderly, alongside with evoking the notions of well-being, quality of life, appreciation and enjoyment of life. Few studies address the conceptualization of the direct use of music in the lives of the elderly. In conclusion, the results confirm the relevance of the theme in health promotion in the elderly population. Also, the results prompts for the need of further investigations related to this practice by health professionals.
\end{abstract}

Key Words: Music; Elderly and health.

\section{LOS EFECTOS DE LA MÚSICA PARA LOS ANCIANOS: REVISIÓN SISTEMÁTICA}

\section{Resumen}

La pesquisa tuvo por objeto trazar el perfil y analizar las publicaciones acerca del uso de la música como herramienta en el cuidado al anciano. La revisión fue realizada en las bases Scielo, Capes y Lilacs a partir del descriptor anciano. Fueron seleccionados artículos publicados en periódicos indexados en el período de 2000 a 2011. A partir de los criterios de inclusión/exclusión fueron recuperados 05 trabajos, en la integra, para discusión. Estos criterios relativos al área de la salud acometen directamente los beneficios que la utilización de la música provoca en la salud y en la vida del anciano, evocando juntamente nociones de bien-estar, cualidad de vida, valorización y placer de vivir. Los trabajos abordan la conceptualización del uso directo de la música en la 
Gomes, Amaral. Os efeitos da utilização da música para os idosos: revisão sistemática

vida del anciano. Concluyendo, los resultados confirman la actualidad y pertinencia del tema para uso en la promoción de la salud en la población anciana, alertando a la necesidad de mayores investigaciones relacionadas a la práctica de tal intervención por los profesionales de la salud.

Palabras-Clave: Música; ancianos y salud.

\section{INTRODUÇÃO}

Conforme dados do Instituto Brasileiro de Geografia e Estatística (IBGE), a população idosa no Brasil vem apontando mudanças na estrutura etária da sociedade brasileira. A representatividade dos grupos populacionais no total, em 2010, era menor que a observada em 2000, para todas as faixas com idade até 25 anos, ao passo que os demais grupos etários aumentaram suas participações na última década. ${ }^{(1)}$

Em 2010, simultaneamente, o alargamento do topo da pirâmide pode ser observado pelo crescimento da participação relativa da população com 65 anos ou mais, que era de 4,8\% em 1991, passando a 5,9\% em 2000 e chegando a 7,4\% em 2010. O crescimento absoluto deste grupo no Brasil, nestes últimos dez anos, se deu principalmente em função do crescimento da comunidade adulta, com destaque também para o aumento da participação de pessoas da terceira idade. Tais mudanças ocorrem devido ao avanço da medicina e as melhorias nas condições gerais de vida da sociedade o que repercutem no sentido de elevar a média de vida do brasileiro que era de 45,5 anos de idade em 1940, para 72,7 anos em 2008, ou seja, mais 27,2 anos de vida. ${ }^{(1)}$

O envelhecimento é caracterizado por mudanças morfofuncionais ao longo da vida, que ocorrem após a maturação sexual e que, progressivamente, compromete a capacidade de resposta dos indivíduos ao estresse ambiental e à manutenção da homeostasia. Entretanto, as transformações presentes nesta fase da vida também são de natureza psicológica, emocional, social e espiritual. ${ }^{(2)}$

A longevidade apesar de demonstrar um avanço da tecnociência, assim como, dos padrões de vida da população, traz consigo a presença de enfermidades não transmissíveis e progressivas, resultando por consequência em incapacidades e fragilidades. Esta realidade tem levado a uma reorganização do Sistema Social e da Saúde, pois a presença deste perfil epidemiológico acarreta consigo um aumento nos gastos públicos em resposta a uma demanda diferenciada do que era visto na década de $50 .^{(1)}$

Nesta perspectiva observa-se na atualidade o surgimento de pesquisas sobre novas intervenções terapêuticas, preventivas e de reabilitação no intuito de promover o 
envelhecimento ativo e funcional, proporcionando, por conseguinte, qualidade de vida. Estas investigações vão de encontro a Política Nacional de Saúde da Pessoa Idosa (PNSPI) a qual foi aprovado em 19 de outubro de 2006, através da portaria $n^{\circ} 2.528$. A PNSPI tem por finalidade recuperar, manter e promover a autonomia e a independência do idoso, através de medidas coletivas e individuais de saúde. ${ }^{(3)}$

Dentre as várias ações de promoção e prevenção de saúde, destaca-se: a aplicação de vacinas - prática recente para idosos -, programas educacionais, atividades físicas, grupos de convivência, além de ações dirigidas à detecção precoce de enfermidades não-transmissíveis, antecipação de danos sensoriais, protocolos para risco de queda, alterações do humor, perdas cognitivas, prevenção de perdas dentárias, prevenção em deficiência nutricional, prevenção da perda de independência, autonomia e prevenção de isolamento social, criando para este grupo oportunidades de fazer parte ou uso de clubes, centros de convivência, associações onde estes artifícios são fundamentais para o idoso continuar inserido na sociedade. ${ }^{(4)}$

Os tratamentos alternativos, ou seja, as terapias complementares e de cuidado à saúde não são presentemente consideradas parte da medicina convencional. Estas práticas estão obtendo reconhecimento da população e da sociedade formal quanto à experiência com o processo adoecimento-cuidado-cura e reequilíbrio do paciente nos momentos atuais. ${ }^{(5)}$

A procura por estas terapias complementares possui maior relevância nos países pobres devido a adequação cultural, fácil acesso da população, quando comparada a dificuldade do acesso à saúde hospitalar e o custo elevado de um atendimento digno nesses países. Os tratamentos complementares possuem um grande enfoque nas ações de promoção da saúde, buscando valorizar estratégias de resgate ao aumento da autoestima, capacidade de adaptação ao meio e melhoria na qualidade de vida. ${ }^{(5)}$

É possível observar que hoje já existe um grande avanço do uso de práticas integrativas e complementares no tratamento de reabilitação na saúde da população, dentre elas: meditações, yoga, hidroginástica, massagens, acunpuntura e música. Ações estas que trazem grandes benefícios à saúde do individuo, contribuindo de forma positiva na promoção da saúde. ${ }^{(6)}$

Salientando uma das terapias integrativas e complementares mais usadas na atualidade, destaca-se a música como um recurso para realização de ações de promoção e prevenção. Na área da geriatria e gerontologia, o uso da música vem se sobressaindo por proporcionar efeitos significativos nas esferas psicoemocionais, físicas e sociais destas pessoas, repercutindo na melhora da autoestima e da sociabilização. 
Gomes, Amaral. Os efeitos da utilização da música para os idosos: revisão sistemática

Nesta perspectiva, esta pesquisa teve como objetivo traçar o perfil e analisar as publicações acerca do uso da música como ferramenta no cuidado à pessoa idosa.

Desta forma, a iniciativa de abordar o tema, os efeitos da utilização da música para idosos incidiram a partir de inquietações, experiências e vivências no decorrer do curso de Enfermagem. Isto através de estudos, discussões, atividades desenvolvidas e observadas por meio de relatos de idosos sobre o uso da música.

\section{METODOLOGIA}

Este trabalho é fruto de uma revisão sistemática. Esta por sua vez, é um recurso importante para elaboração da prática baseada em evidências que consiste em uma forma de síntese dos resultados de análises relacionadas com um problema específico. ${ }^{(7,8)}$

A pesquisa bibliográfica abordando a temática teve início através de um levantamento de artigos publicados em periódicos localizados nas bases de dados on line: Scientific Eletronic Library Online (Scielo) Coordenação de Aperfeiçoamento de Pessoal de Nível Superior (Capes) e Literatura Latino-Americana e do Caribe em Ciências da Saúde (Lilacs). Utilizaram-se como palavra-chave: "música e idoso", "envelhecimento e saúde", "musicoterapia e velhice". A seleção preencheu os seguintes critérios: ter sido publicado na língua portuguesa, texto na íntegra, ter como autores profissionais de saúde, musicoterapeutas e educadores físicos, publicação entre os anos de 2000 a 2011.

Foram identificados 27 artigos publicados nas bases de dados determinada, destes 22 foram recuperados no levantamento inicial através de leituras exploratórias de seus resumos. Dos 22 artigos recuperados, 03 foram excluídos por não estarem disponíveis na íntegra gratuitamente e 14 não contemplavam o objetivo do estudo proposto. Por fim, para realização do levantamento bibliográfico foram identificados apenas 05 artigos que abordam o uso da música no contexto da saúde da pessoa idosa.

Nas pesquisas foram adotados critérios de inclusão e exclusão de artigos, onde foram considerados como objetos de estudos para revisão de textos aqueles que falam diretamente sobre a influência e benefícios do uso da música na vida do idoso. Portanto, no final da pesquisa, foi realizada uma leitura interpretativa dos teóricos, buscando analisar de forma mais ampla e analítica os conhecimentos.

Dessa forma, os trabalhos analisados referem-se à melhoria que o uso da música trás na vida do idoso como terapia, além de apresentar também as mudanças que a mesma 
influência nos fatores físicos, emocionais, sociais que influem diretamente na qualidade de vida destes.

Observaram-se ainda, outras produções com o mesmo propósito, porém não constituía propriamente a categoria que esta em estudo, sendo estes excluídos da análise, são eles: atividade física e bem-estar (01 trabalho) dança (07 trabalhos), percepção dos profissionais (03 trabalhos), canto (01 trabalho), arteterapeutica (01 trabalho).

Outrora, optamos por fazer uso dessas 05 referencias, por possuir características e elementos em comum que desencadeiam o processo da terapia.

\section{RESULTADO \& DISCUSSÃO}

Os resultados foram divididos em duas fases, na primeira foi apresentado o perfil dos artigos publicados e selecionados. Enquanto, que na segunda fase foi realizada uma análise das ideias centrais dos mesmos. Para tanto, classificou-se os estudos em duas categorias denominadas de: "Terapia musical como melhoria na qualidade de vida "física" e "Terapia musical como melhoria da qualidade de vida psíquica-autoestima".

$\mathrm{Na}$ primeira fase os resultados e discussão foram apresentados em forma de tabela para melhor entendimento e visualização dos dados obtidos seguidos das discussões.

\begin{tabular}{|l|l|c|}
\hline \multicolumn{1}{|c|}{ Autores } & \multicolumn{1}{|c|}{ Título do Artigo } & $\begin{array}{l}\text { Ano de } \\
\text { Publicação }\end{array}$ \\
\hline NOORDHOEK J; JOKL L. & $\begin{array}{l}\text { Efeito da música e de exercícios físicos } \\
\text { num grupo de pessoas reumáticas: estudo } \\
\text { de caso. }\end{array}$ & 2008 \\
\hline CÔRTE B; LODOVICI NETO P. & A musicoterapia na doença de Parkinson. & 2009 \\
\hline SOUZA SL. & Educação Musical com Idosos. & 2005 \\
\hline LEÃO ER; FLUSSER V. & $\begin{array}{l}\text { Música para idosos institucionalizados: } \\
\text { percepção dos músicos atuantes. }\end{array}$ & 2008 \\
\hline MIRANDA MLJ; GODELI MRCS & $\begin{array}{l}\text { Avaliação de idosos sobre o papel e a } \\
\text { influência da música na atividade física. }\end{array}$ & 2002 \\
\hline
\end{tabular}

Quadro 1 - Distribuição dos títulos segundo os autores e o ano

Fonte: elaborada pelas autoras com base em dados bibliográficos, 2011. 
$\mathrm{Na}$ seleção empreendida no presente estudo, as publicações científicas estão concentradas no ano de 2002 a 2009, o que mostra a atualidade do tema e sua circulação científica recente.

\begin{tabular}{|l|c|l|}
\hline \multicolumn{1}{|c|}{ Autores } & Ano & \multicolumn{1}{c|}{ Categoria Profissional } \\
\hline NOORDHOEK J; JOKL L. & 2008 & Terapeuta Ocupacional e Farmacêutica. \\
\hline CÔRTE B; LODOVICI NETO P. & 2009 & $\begin{array}{l}\text { Musicoterapeuta e Pós Graduada em } \\
\text { Gerontologia e Antropólogo. }\end{array}$ \\
\hline SOUZA SL. & 2005 & $\begin{array}{l}\text { Professora de música e especialista em } \\
\text { geriatria e gerontologia. }\end{array}$ \\
\hline LEÃO ER; FLUSSER V. & 2008 & Enfermeira e Musicoterapeuta. \\
\hline MIRANDA MLJ; GODELI MRCS. & 2002 & Educador (a) Físico. \\
\hline
\end{tabular}

Quadro 2 - Distribuição dos artigos segundo os autores, ano e categoria profissional

Fonte: elaborada pela autora com base em dados bibliográficos, 2011.

No Quadro 2, é possível observar que nos estudos analisados foram identificas várias categorias profissionais envolvidas no estudo sobre a música como terapia na qualidade de vida do indivíduo. Isto representa um resultado satisfatório do estudo, onde é possível identificar que o processo da terapia não convencional é também de interesse multidisciplinar para os profissionais da saúde. O mesmo contribui para sua inserção no meio em que se atua e o fortalecimento como tratamento positivo na população estudada.

É de extrema importância que exista um maior enfoque e estudo a respeito do benefício que a música traz aos pacientes.

\begin{tabular}{|l|c|l|}
\hline \multicolumn{1}{|c|}{ Autores } & Ano & \multicolumn{1}{c|}{ Metodologia } \\
\hline NOORDHOEK J; JOKL L. & 2008 & $\begin{array}{l}\text { Sessões de música e breve entrevista com } \\
\text { os pacientes. }\end{array}$ \\
\hline CÔRTE B; LODOVICI NETO P & 2009 & $\begin{array}{l}\text { Entrevista pré-estrututarada e } \\
\text { reestruturada. }\end{array}$ \\
\hline SOUZA SL. & 2005 & $\begin{array}{l}\text { Análise da literatura a respeito do } \\
\text { benefício da música para os idosos. }\end{array}$ \\
\hline LEÃO ER; FLUSSER V. & 2008 & $\begin{array}{l}\text { Questionário e o discurso do sujeito } \\
\text { coletivo. }\end{array}$ \\
\hline MIRANDA MLJ; GODELI MRCS & 2002 & $\begin{array}{l}\text { Entrevista semi-estruturada com idosos } \\
\text { de idade média 68 anos. }\end{array}$ \\
\hline
\end{tabular}

Quadro 3 - Distribuição dos artigos segundo autores, ano e metodologia

Fonte: elaborada pelas autoras com base em dados bibliográficos, 2011. 
No Quadro 3, descrita acima, foi apresentada a metodologia utilizada pelos autores na descrição dos trabalhos analisados, compreendendo os meios que estes empregam na comunidade idosa e nos resultados a respeito do benefício que a música traz no cotidiano da mesma.

É notório que dos 05 estudos analisados, a entrevista foi o objeto de análise com maior relevância, aplicada pelos pesquisadores na população estudada para obter a opinião destes idosos a respeito da música. Seguido do uso de questionário e discurso através de vídeo ou gravações. O que se atenta para que exista maior investimento em pesquisa de campo sob o tema a ser observado. Percebendo assim, que só 01 estudo foi feito através da análise de literatura, onde o mesmo relatava a música como processo de educação musical para os idosos. Expondo assim os benefícios que a dinâmica oferecia na melhoria da qualidade de vida.

\begin{tabular}{|l|c|l|}
\hline \multicolumn{1}{|c|}{ Autores } & Ano & \multicolumn{1}{c|}{ População investigada } \\
\hline NOORDHOEK J; JOKL L. & 2008 & Idosos portadores de doenças reumáticas. \\
\hline CÔRTE B; LODOVICI NETO P. & 2009 & $\begin{array}{l}\text { Idosos portadores de Doença de } \\
\text { Parkinson. }\end{array}$ \\
\hline SOUZA SL. & 2005 & Idosos de uma escola de música. \\
\hline LEÃO ER; FLUSSER V. & 2008 & Idosos institucionalizados. \\
\hline MIRANDA MLJ; GODELI MRCS & 2002 & $\begin{array}{l}\text { Idosos ativos que fazem parte do } \\
\text { programa: autonomia para a atividade } \\
\text { física. }\end{array}$ \\
\hline
\end{tabular}

Quadro 4 - Distribuição dos artigos segundo autores, ano e população investigada

Fonte: elaborada pelas autoras com base em dados bibliográficos, 2011.

Em relação à população pesquisada, nos estudos específicos sobre a utilização da música, pôde-se observar, no Quadro 4, que as pesquisas incluídas focalizam a pessoa idosa, não trazendo a percepção da família e dos profissionais de saúde.

Contudo, devido ao número restrito de trabalhos selecionados foi preciso relativizar esse aspecto, embora se deva salientar a escassez de produtividade constatada, o que confere relevo ao tema e confirma a necessidade de maior estudo sobre o assunto para prática clinica na área de promoção, prevenção e reabilitação da saúde. 
Gomes, Amaral. Os efeitos da utilização da música para os idosos: revisão sistemática

No entanto, como o foco da revisão sistemática é, também, analisar o uso da música na atenção à saúde do idoso, de forma subjetiva e modo amplo, dar-se-á inicio a segunda fase da pesquisa que representa uma análise dos resultados dos artigos selecionados.

\section{CATEGORIA 1: TERAPIA MUSICAL COMO MELHORIA NA QUALIDADE DE VIDA "FÍSICA"}

Esta categoria foi criada em virtude dos estudos selecionados que apresentam como repercussão da terapia musical apresentada a melhora na qualidade de vida 'ffísica' das pessoas idosas investigadas. Assim, segue discussão sobre os principais pontos destacados pelos autores dos 02 trabalhos que contemplam o primeiro eixo temático. São eles: Noordhoek e Jokl ${ }^{(9)}$ e Côrte e Lodovici Neto. ${ }^{(10)}$

É evidente em algumas análises estudadas, que a música é algo que sopra ao ser humano como relaxamento e bem estar. Isso é evidente nas revisões feitas, os quais mostram o benefício que a música traz para a vida do ser humano, este que vive em uma sociedade de estresse e sofrimento.

A partir disso, pode-se analisar que a música é sugerida pelos profissionais de saúde como ações terapêuticas, preventivas e de reabilitação. Já que esta foi observada como resultado positivo na melhoria da qualidade de vida dos idosos e na recuperação de patologias. ${ }^{(9)}$

Côrte e Lodovici Neto ${ }^{(10)}$ consideram que a produção científica atual, acerca do tema, procura compreender a importância de tal terapia que leva à melhoria da saúde, sob perspectiva positiva. Estes trazem o tratamento como ação que minimiza o sofrimento, o que implica em uma melhor relação com si e com quem o cerca. Além, de tentar mostrar que o doente pode restabelecer-se e manter ou voltar a ter contato com o mundo, numa aproximação com a realidade, estabelecendo laços afetivos, a ponto de não se centrar só na doença minimizando seus efeitos, o que pode reverter de forma positiva em sua subjetividade.

Côrte e Lodovici Neto ${ }^{(10)}$ mostram ainda que o tratamento de pessoas com doenças crônicas em uso da música acontece por esta terapia ser algo que afeta todo o cérebro. Nas suas experiências relatadas com os pacientes foi possível perceber que alguns idosos que apresentavam acinesia unilateral ao ouvir música conseguiram fazer com que o seu corpo funcionasse em perfeita união. Estes autores ${ }^{(10)}$ reforçam também a importância da música em 
diversos problemas neurológicos ao citar Sacks quando o mesmo revela que "a música a liberta da doença por algum tempo - e não só a música, mas a imaginação da música".

É certo que a música é essencial aos pacientes de doenças crônicas, já que esta faz com que os mesmos se sintam bem, livres, independentes e vivos. A música quando ouvida é como se todos os sintomas existentes neste paciente desaparecessem.

As experiências de Sacks com seus pacientes no estudo apresentado por Côrte e Lodovici Neto ${ }^{(10)}$ informam que a música faz uma excelente via de tratamento do doente, fazendo com que este possa conviver melhor com a doença, tal terapia traz a estes indivíduos melhor reabilitação minimizando seu sofrimento. Estas práticas terapêuticas vêm trazendo resultados positivos no tratamento das patologias, que afetam a capacidade física, cognitiva, psicológica ou subjetiva das pessoas.

Analisando ainda sobre os aspectos físicos positivos que a música traz como intervenção na vida dos idosos, fica ainda mais evidente que esta terapia funciona na melhoria da qualidade de vida destes. No estudo de Noordhoek e Jokl, ${ }^{(9)}$ pode-se considerar que a interação dessas áreas terapêuticas não só é possível, mas, sobretudo, positiva. Já que esta interfere ao mesmo tempo, nos aspectos emocionais, físicos e sociais da população analisada. Segundo Noordhoek e Jokl, ${ }^{(9)}$ isto é percebido no momento em que o paciente perde a vontade de viver e a depressão toma conta da sua vida.

Neste contexto complexo entra a música com o papel de mudança na vida destas pessoas, permitindo que estes expressem suas emoções e sentimentos e possam enxergar "caminhos" possíveis para aceitação do problema e vontade de aceitação do tratamento, tendo como objetivo maior fornecer meios que auxiliem na superação dos desafios impostos pela patologia acometida. ${ }^{(9)}$

Na mesma análise é possível enxergar que a interação da terapia com música, junto à realização de exercícios físicos tem revelado que tal associação não é apenas possível, mas, sobretudo, importante pela dimensão existencial que esta alcança na vida do indivíduo.

A música interfere de forma direta na recuperação da memória e torna-se assim, uma terapia auto-expressiva e de atuação relevante como modelo de prevenção, reabilitação e intervenção na vida destas pessoas. ${ }^{(10)}$ 
CATEGORIA 2: TERAPIA MUSICAL COMO MELHORIA NA QUALIDADE DE VIDA "PSÍQUICA" COM RELAÇÃO À AUTO-ESTIMA

O segundo eixo temático de maior expressão está representado por produções que apresentam revisão sistemática sobre música, idoso, saúde, totalizando 03 trabalhos. São artigos que abordam o conceito de terapia musical como melhoria da qualidade de vida "psíquica", com certa proximidade a "autoestima", as quais guarda certa proximidade conceitual com o bem-estar psicológico e da saúde. São eles: Souza, ${ }^{(11)}$ Leão e Flusser ${ }^{(12)}$ e Miranda e Godeli. ${ }^{(13)}$

Nesta análise dos estudos selecionados, os autores apresentam os resultados de uma investigação que tem por objetivo determinar como a terapia musical contribui na melhoria da qualidade de vida "psíquica", relacionando a "autoestima", ou seja, relação da música com o comportamento do idoso. ${ }^{(12)}$

O alvo da pesquisa foi uma população de idosos que usa a música como propósito de satisfação na vida, melhora comportamental e psicológica, bem como as necessidades de enriquecimento na autoestima relacionada à idade, perda física que podem estar correlacionadas. $^{(13)}$

Segundo os resultados da observação, o bem estar apresentou forte correlação com o uso da música nas atividades exercidas pelos idosos, a autoestima apresenta forte correlação com a satisfação de viver. ${ }^{(13)}$

Deve-se considerar que as evidências disponíveis se afirmam e, que o uso da música é um sistema dinâmico que opera de diversos modos, afetando dimensões como o comportamento social. Por esse prisma, a música, deveria ser investigada dentro de um cenário específico, em um processo contínuo e constante, considerando seu dinamismo peculiar. $^{(11)}$

$\mathrm{Na}$ análise de seu estudo é possível também perceber que a mesma identifica que o "aprendizado musical pode contribuir para o processo e para uma melhor qualidade de vida". (11)

Segundo Côrte e Lodovici Neto ${ }^{(10)}$ a musicoterapia é o que tem de mais essencial nos processos terapêuticos, levando a pessoa afetada por uma doença a manter uma posição "resiliente" diante da vida, minimizando a sintomatologia e transformando a si mesma, ganhando força para estancar sua progressão. Tudo isto porque a música possibilita que a 
pessoa orquestre a mente, corpo, alma e coração resgatando sua identidade e fazendo com que o indivíduo se torne maestro da sua própria vida.

As literaturas relatam que o aumento do tratamento com música na terceira idade estimula, além do prazer de cantar, tocar, improvisar, criar e recriar musicalmente, o redescobrir das canções que fizeram e fazem parte da sua vida sonoro-musical. As pesquisas mostram que estes tratamentos auxiliam diretamente no resgate da identidade sonora do cliente, tendo por consequência a elevação do seu amor próprio e autoconfiança. Com esta população este tratamento vem se mostrando de grande importância, no que se refere aos resgates de memória, como tratamento coadjuvante de valor reconhecido mundialmente, já que sua eficácia na manutenção das funções cognitivas, elevação da autoestima e sociabilização. ${ }^{(11)}$

Na terceira idade, a música representa uma terapia auto-expressiva e de grande atuação nas funções cognitivas, a qual pode ser estimulada pelo canal sonoro-musical, onde as instâncias psíquicas muitas vezes não poderão alcançar as palavras. Ou seja, em instâncias mentais nas quais a linguagem verbal, devido ao acometimento por doenças e deficiências, já não intervém com grande poder de penetração. ${ }^{(10)}$

No tratamento do uso da música como terapia é comum observar no idoso a emergência de se elaborar conteúdos e fortalecer os mesmos, nesta produção se constata a reestruturação do individuo, sujeito de suas próprias ações e fortalecimento de sua identidade. Todo este processo existe porque a música acompanhará o processo de envelhecimento, marcando as épocas e os acontecimentos sociais. Ao marcar um tempo, a canção, por seu vínculo afetivo, pode resgatar o fio melódico da vida do individuo, ao retratar todas as suas idades no contexto sonoro musical. Tais lembranças vinculam as vivências pessoais e intransferíveis às vivências sociais e coletivas. Com este processo o idoso se vê restituído, a partir de sua própria produção e ação funções vivo que, devido ao processo natural do envelhecimento, foram se alterando com o tempo, ou que foram alteradas por algum processo patológico. ${ }^{(10)}$

Em um deles nota-se que mesmo com as mudanças na sociedade atual, é preciso repensar o que se deseja para que se possa chegar ao envelhecimento com uma boa qualidade de vida. Assim, se faz necessário a busca de ações que promovam o envelhecimento saudável que resultará numa vida mais tranquila. ${ }^{(11)}$

Para concluir as idéias analisadas no estudo de revisão no estudo de Leão e Flusser ${ }^{(12)}$ estes dizem que a música possibilita a inserção do idoso em contextos sociais, carregados de 
atividades significativas que o leve a pensar e a querer, o que intensifica a atividade diencefálica, a mesma traz que esta experiência minimiza o isolamento social. E sua importância para a promoção, prevenção e reabilitação das mesmas, possibilitando assim uma melhor qualidade de vida.

Assim, constata-se a necessidade de maior estudo sobre o assunto devido à carência, tanto no nível nacional quanto no contexto internacional, o que aponta para construção de aperfeiçoamento do campo já existente.

\section{CONSIDERAÇÕES FINAIS}

De modo geral, nos limites da análise efetuada, pode-se afirmar que a maioria dos trabalhos revisados situa-se dentro da perspectiva da promoção da saúde.

Os estudos específicos acerca do uso da música na velhice ainda não se destacam de modo expressivo, na configuração do campo delineado por esta revisão. O movimento percebido, a partir da análise do panorama oferecido pela literatura, é que a produção científica atual, muito mais do que discutir a noção de música como terapia,estuda os problemas envolvidos em sua definição e os pressupostos teórico-conceituais que a sustentam, preocupa-se com o modo como o conceito pode ou deve ser aplicado à prática clínica, mais especificamente na promoção, prevenção e reabilitação da saúde.

Por sua vez, a atenção é voltada para delimitar em que medida uma determinada técnica ou intervenção pode contribuir para a melhoria do bem-estar das pessoas, o que implica diretamente no conceito de qualidade de vida e, consequentemente de saúde.

Em relação á definição do uso da música os estudos levantados apresentam seus contornos conceituais de modo bastante similar. Essa noção, no entanto, é permeada por outros conceitos que o tangenciam como o de satisfação de vida, bem-estar psicológico e qualidade de vida. Todas essas noções contempladas pelos estudos apesar de estarem intimamente relacionadas, estes são diferentes e deve ser considerado em suas nuanças e especificidades, o que não ocorre na maioria das investigações.

Nesse sentido, são necessários estudos futuros que problematizem a possível dissociabilidade semântica entre esses conceitos quando se pensa e se investiga qualidade de vida.

Como se constatou nos resultados apresentados, diversos trabalhos incluídos nesta revisão sistemática estabeleceram correlações entre as variáveis de música, terapia e outro 
contexto como idade, cultura, promoção da saúde, qualidade de vida, bem estar, prevenção e reabilitação da saúde. Tais estudos também têm impacto sobre a definição do conceito do uso da música, na medida em que auxiliam a hipotetizar quais as variáveis que estariam direta ou indiretamente relacionadas a esse construto. Frente à complexidade que o tema evoca, urge a necessidade de maior refinamento teórico-metodologico, além de adequada contextualização dos processos de investigação para sua melhor compreensão.

Pelo que foi explicitado na análise do perfil desenhado pelos estudos incluídos, há a necessidade de aprofundar o conhecimento dos fatores relacionados à música, de modo a propiciar maior densidade teórica para esse campo em construção e maiores investimentos na pesquisa de campo sobre tal problematização.

Por outro lado, a análise empreendida é limitada pela reduzida disponibilidade de estudos publicados e pelas próprias limitações da leitura que deles pode ser feita. De todo modo, esses estudos funcionam como um norte para se conhecer a produção cientifica, embora nem sempre possuam o alcance necessário para se refletir sobre aspectos metodológicos e teóricos da música na qualidade de vida do idoso.

Seria desejável que essas investigações não apenas delimitassem conceitualmente a noção, mas se preocupassem em produzir dados empíricos que contribuíssem para delineamento consistente do conhecimento sobre a utilização da música, nas ações de promoção da saúde e melhoria da qualidade de vida física, psíquica e social desta população.

Desse modo, o profissional de saúde poderia melhor usufruir de tal terapia como referência para pautar sua prática cotidiana.

Essa articulação teórico-prática, em última instância, é necessária para que se possa pensar na aplicabilidade do construto no planejamento de programas de promoção, prevenção e reabilitação, visando à transformação dos modelos e práticas atuais, conforme é almejado um melhor resultado na saúde destes pelos profissionais, que se preocupa com a promoção da saúde, bem como os aspectos salutares e adaptativos do desenvolvimento.

Certamente esse caminho inovador se mostra árduo e complexo, pois nele a abertura para o novo tem que operar concomitantemente com a ruptura e o conhecimento estabelecido, de modo que a mudança não apague o que já foi construído, mas imprima um novo olhar para as formas cristalizadas de se ver e de se conceber o ser humano.

Nesse sentido, a revisão sistemática apresentada é um elo a mais dentro de uma corrente, a partir da qual poderão ser elaborados outros levantamentos bibliográficos e discutir qual o impacto dessas publicações na definição e no estudo científico a respeito da música e 
qualidade de vida, contribuindo com reflexões conceituais e teórico-metodológicas relevantes a respeito do olhar positivo dessa intervenção na saúde da população idosa de forma relevante para o planejamento de novas pesquisas na área. Ressaltando que o resultado benéfico de tal terapia é comprovado por relatos adquiridos na terapia e não uma prova realizada através de estudos por experimentos científicos.

Dessa maneira, será possível delinear novas perspectivas de estudos e intervenções sobre tal tema para que este comece a tomar maior relevância na população adquirida e maior conhecimento na população acadêmica.

\section{REFERÊNCIAS}

\section{INSTITUTO BRASILEIRO DE GEOGRAFIA E ESTATÍSTICA. Censo}

Demográfico 2010. [acesso em 2011 ago 04]. Disponível em:

$<$ http://www.ibge.gov.br/home/presidencia/noticias/noticia_visualiza.php?id_noticia=186 6\&id_pagina $=1>$.

2. Freitas EV, Py L, Cançado FAX, Doll J. Tratado de geriatria e gerontologia. $3^{\text {a }}$ ed. Rio de Janeiro: Editora Guanabara Koogan; 2006. p. 1216.

3. Brasil. Ministerio da Saúde. Portaria MS No 2.528/06. Política Nacional da Saúde da Pessoa Idosa. Brasília, 2006.

4. Brasil. Ministério da Saúde. Estatuto do Idoso. $2^{\mathrm{a}}$ ed. Brasília: Ministério da Saúde; 2006. 70p.

5. Tesser CD. Práticas complementares, racionalidades médicas e promoção da saúde: contribuições poucos exploradas. Cad. Saúde Pública [online]. 2009, vol.25, n.8, p. 17321742

6. Brasil. Ministério da Saúde. Amapá conta com centro de referência para toda a região. Revista Brasileira Saúde da Família. 2008; IX, Edição Especial: p.30-4.

7. Sampaio RF, Mancini MC. Estudos de revisão sistemática: um guia para síntese criteriosa da evidência científica. Rev. bras. fisioter. [online]. 2007, 11(1): 83-9.

8. Galvao CM, Sawada NO; Trevizan MA. Revisão sistemática: recurso que proporciona a incorporação das evidências na prática da enfermagem. Rev. Latino-Am. Enfermagem [online]. 2004; 12(3); 549-556.

9. Noordhoek J, Jokl L. Efeito da música e de exercícios físicos num grupo de pessoas reumáticas: estudo piloto. Acta fisiátrica. 2008; 15(2): 127-129.

10. Côrte B, Lodovici Neto P. A musicoterapia na doença de Parkinson. Ciênc. saúde coletiva [online]. 2009;14(6): 2295-2304.

11.Souza SL. Educação musical com idosos. Textos Envelhecimento [online]. 2005; 8(3): 411-427. 
Gomes, Amaral. Os efeitos da utilização da música para os idosos: revisão sistemática

12.Leão ER; Flusser V. Música para idosos institucionalizados: percepção dos músicos atuantes. Rev. Esc. Enferm. USP. 2008; 42(1):73-80.

13.Miranda MLJ, Godeli, MR. Avaliação de idosos sobre o papel e a influência da música na atividade física. Revista Paulista de Educação Física. 2002; 16(1): 86-99. 